\title{
References
}

Chandrasekhar, S. and Limber, 'D. N.: 1954, Astrophys. J. 119, 10.

Ferraro, V. C. A.: 1954, Astrophys. J. 119, 407.

Goossens, M.: 1972, Astrophys. Space Sci. 16, 386 (Paper I).

Goossens, M., Smeyers, P., and Denis, J.: 1976, Astrophys. Space Sci. 39, 257 (Paper II).

Prendergast, K. H.: 1956, Astrophys.J. 123, 498.

Prendergast, K. H.: 1958, Astrophys. J. 128, 361.

Singh, S. and Tandon, J. N.: 1969, Astron. Astrophys. 2, 266.

Tassoul, J. L.: 1968, Monthly Notices Roy. Astron. Soc. 138, 123.

Wentzel, D. G.: 1962, Astrophys. J. 135, 593.

Woltjer, L.: 1962, Astrophys. J. 135, 235.

\section{Erratum to Paper II}

(Astrophysics and Space Science, 39, 257)

The right-hand member of Equation (49) of this paper should read as follows:

$$
\begin{aligned}
& \frac{8}{2} \frac{8}{5} \pi^{2} G \varrho^{2} R^{5}\left\{\sum_{k} \frac{N_{k, m}}{k+1} \Delta_{k}^{2}+\frac{b(R)}{R}\left(2 \frac{b(R)}{R}-\frac{\mathrm{d} b}{\mathrm{~d} R}\right) \times\right. \\
& \times\left[8 F_{l, m}-\frac{4}{3} l(l+1)\left(2 F_{l, m}+N_{l, m}\right)\right]- \\
& -\frac{2}{3} \frac{a(R)}{R}\left(2 \frac{a(R)}{R}-\frac{\mathrm{d} a}{\mathrm{~d} R}\right)\left(N_{l, m}-F_{l, m}\right)- \\
& -2 F_{l, m}\left[\frac{a(R)}{R}\left(2 \frac{b(R)}{R}-\frac{\mathrm{d} b}{\mathrm{~d} R}\right)+\frac{b(R)}{R}\left(2 \frac{a(R)}{R}-\frac{\mathrm{d} a}{\mathrm{~d} R}\right)\right]+ \\
& \left.+m^{2} N_{l, m} \frac{b(R)}{R}\left(2 \frac{b(R)}{R}-\frac{a(R)}{R}\right)\right\} .
\end{aligned}
$$

As the numerical computations have been done with a correct expression for the right-hand member of Equation (49), the numerical results presented in Paper II are correct. 